\title{
Certified Registered Nurse
}

National Cancer Institute

\section{Source}

National Cancer Institute. Certified Registered Nurse. NCI Thesaurus. Code C71332.

A reg istered nurse who has received certification from a national nursing org anization or national nurse certification org anization for practice in a specialized field of professional nursing. 